\title{
Application Study on Soft Decoration Materials in Modern Living Space
}

\author{
Jie Li \\ Institute of Art \& Design, Nanchang Hangkong University, Nanchang, China
}

\begin{abstract}
Modern housing is not only a concept to satisfy the demands of "residence". Human beings have started to expand and extend toward the essence of living. As a way of emotional sustenance and expression for modern people, soft decoration has been gaining its popularity in the industry of soft decoration quietly. Residents' requirements and attitudes toward their dwelling environment have led to the qualitatively leaping development of interior design, such a young industry. Problems including how to better recognize the concept, design requirements and demands of soft decoration and the morphological difference of decoration and being decorated and how does software decoration offer new vitality to interior space are to be considered by modern interior designers. This paper conducts an analysis on the application of soft decoration in modern interior design and the development of soft decoration in modern interior design.
\end{abstract}

Keywords-soft decoration; modern interior design; culture

\section{INTRODUCTION}

Interior design is the extension and development of architectural design. Along with social progress and the development of human civilization, interior design can't just satisfy the functional demands, but also becoming the important content of architectural design and environment design, and also the core for sustainable architectural development. In a manner of speaking, interior design has turned into an independently emerging discipline that demonstrates integrity. "Soft decoration" in interior design is just like the warm sunshine in winter that nourishes people. Interior design can integrate hard decoration into the interior space through soft decoration; use the design language to influence the entire effect and promote the further sublimation of taste and connotation in decoration uniformly. Soft decoration of interior design can effectively strengthen the style of interior design, rapidly create the interior atmosphere, demonstrate the personalized demands and create the diversified and plentiful quadratic space.

\section{DEFINITION OF SOFT DECORATION}

As the name implies, "soft decoration" is a concept in interior design that is opposite to fixed interface decoration and functional decoration within architectures. In narrow sense, soft decoration in interior design indicates all of objects made of soft materials, dominated by interior fabrics. However, this semantic category has been broken long ago. In wide sense, "soft decoration" indicates all of movable and replaceable decorations and all of behaviors that can improve interior functions foil the atmosphere and demonstrate interior culture, except fixed decorations, including wall modeling, floor pavement and suspended ceiling. It not only includes those decorations for interior design, but also is a kind of design thinking and method, paying more attention to unification of humanistic functional design and art taste through decoration design. "Soft decoration" can improve spatial order and structure and integrate the hard and soft decoration to help people experience what is returning to origin.

Traditionally, residential accessory indicates the auxiliary design for sublimation to strengthen the style effect, after room space and functions have been divided and basic decoration to ensure the residential use has been completed. Today, soft decoration has become a comprehensive discipline crossing architecture decoration, fashion, life and art. The existence reason and development direction of soft decoration is to demonstrate the confidence of clients and display their unique styles and effects. All of accessories and furniture involved in this process are in wide range, including fabrics, sofa, tea table, table, TV bench, decorative picture, decorative lighting, green plant and handicraft. From the perspective of development, people have better understanding and higher visual pursuit of decoration design and pay more attention to realizing the expected effect of furnishings through furniture and accessories, meaning the transformation of public values that drive the development of architecture decoration industry in China.

\section{FUnCTION OF SOFT DECORATION IN MODERN INTERIOR DESIGN}

From the perspective of living environment, soft decoration can use different elements to display different spatial characters and different cultural connotations, to transfer the single, plain and bull static space into a temperamental and interesting dynamic space of multiple levels that can reveal the owners' cultural accomplishment, aesthetic values, life experience and even internal emotions.

Firstly, an excellent interior design that can display the details of daily life depends on soft decoration. Usually, when we visit an interior space, we will find that the most attracting place may not be the exquisite cabinet or huge, but those movable or replaceable curtains, carpets, a piece of painting or china bottle. Those soft decorations can reveal the masters' emotional elements. Soft decoration, just like dressing, can dress for the interior space to endow it with different effects, the charming grace of a modestly cautious gentleman or the extremely luxury noble atmosphere. All of public space designs for homes or hotels rely on soft decorations creating the sentiment, in order to better satisfy people's psychological 
demands. For instance, to create a bedroom space, it will involve curtains, bedding products and carpets, the colors of those soft decoration matters will determine the style characters of the entire room. Meanwhile, some exquisite back cushions and throw pillows act as the highlights of the whole room, the colors of which can enhance the room. Of course, the accessories of shadows, plants and flowers are indispensable to decorate a bedroom. Therefore, soft decoration shall be healthy and environmental-friendly.

Secondly, health is the foundation of human beings; and the increasing concern of design concepts including green environmental protection and energy saving and emission reduction have been concerned increasingly has reflected people's urgent demands on the one hand, and that various materials have been unable to satisfy people's demands, along with scientific development and the in-depth research on ergonomics. However, the interior soft decoration of ecoenvironment protection firstly uses the eco-environment protection of materials and craftsmanship and the energy saving and emission reduction of equipment facilities, e.g. the non-toxic and non-pollution decoration materials and craftsmanship, solar water heater, solar power generation, energy-saving sanitary outfits and energy-saving electrical equipments; secondly applies the design concepts beneficial to our emotional and physical health, e.g. a interior design with recreational and leisure function can undoubtedly provide users with a relaxed environment for working and living; a cute and soft sofa with cartoon image and warm tone and cozy fabric art decoration can create the psychological comfort and pleasure. Thirdly, soft decoration takes the service target and audience into consideration. For instance, a hotel design shall firstly provide customers with a warm and comfortable bed; while a bar design shall pay more attention to bright color contrast and personalized fabric supplement to create the relaxed and free atmosphere. In addition, the consideration of the special characters of elderly, women and children in soft interior decoration has demonstrated its emotional caring. [2]. Fourthly, soft decoration stresses the expression of humanity and emotion through symbol marks. For instance, a wide and luxuriously decorated chair in a study room can stand for identity and status; while the rational hanging and setting of calligraphies and paintings can create the gentle and cultivated environment and atmosphere.

Thirdly, soft decoration, as an important part in interior decoration, plays a significance role. The application of soft decoration can not only bring about the visual shock, but also demonstrate personalized and art pursuit. The diversification of materials, colors and forms of soft decoration has led to its unlimited development space, imagination space and play space. After the basic interior hard decoration, the supplement of soft decoration is of great importance, because soft decoration can adjust the interior color for space beautification, personalization pursuit and emotion expression, which are problems that shall be considered by each designer in soft and hard interior decoration. Soft decoration, based on its diversified materials, colors and patterns, plays the unique role in interior decoration. Soft decoration can also adjust people's visual sense. Through hue contrast and tone application, soft decoration can play the psychological effect on interior space and temperature. For instance, the textures of linen, silk, rattan or bamboo weaving can be achieved through cutting, weaving and knitting. Texture contrast includes the textures of materials, but also the harmonization of light and color. Under the influence of color and light, different materials and their surface textures can provide different visual effects. In a manner of speaking, soft decoration is the indispensably finishing touch for the harmonization of interior decoration color.

Finally, soft decoration can create green environment. Close to nature is the universal property of human beings. Especially when you return home from the noisy city, a small area of oasis can make you spontaneously immersed in relaxation and recreation rapidly. An interior space with rationally allocated plants and flowers or natural environment can be greatly helpful for psychological adjustment and health promotion. Meanwhile, plants and flowers and bonsais can reduce toxically harmful gases in interior space and adjust the interior air quality. Therefore, putting a plant in an appropriate place in the interior space can not only strengthen the space sentiment, but also coordinate the spatial artistry and sense of depth, as the key role of soft decoration. In addition, there are also lighting decoration, linear decoration and furnishing decoration in interior soft decoration, which are necessary ways of expression in interior design. The rational combination of those methods can not only beautify the interior space and satisfy peoples' material and spiritual demands, but also demonstrate people's life values and aesthetic pursuits.

\section{Advantages of ApPlying Soft DeCORATION IN INTERIOR DESIGN}

Firstly, the rational application of soft decoration in interior design can create the diversified and rich quadratic spaces to divide the entire space. The function setting and adjustment is the terminal part in interior decoration. Architectural space is the linear building of the interior structure, which can't be moved or changed in a large scale as the hard condition. If a designer wants to change the floor, wall or ceiling of a room, which are the 3D skeleton and linear space of the room, he/she shall change the original framework. And it has been extended to the quadratic space design. Soft decoration can freely build up the quadratic space without breaking the original linear space. Actually, the application of soft decoration can solve the problem of space division easily. [1] We can use accessories of soft decoration to divide the interior space for rational functions and rich sense of levels.

Secondly, the individualization of soft decoration can demonstrate people's humanity and personality. Soft decoration can display the continuously upgrade concept of designers and residents in interior decoration. The requirements on residential environment has been transmitted from luxury pursuit to the level of natural admiring, concerning culture, personality and expecting value regression gradually. Soft decoration features its unique advantages, e.g. diversified properties, easiness for replacement and laundry, versatile textures and convenience for arrangement. Different soft decorations can appropriately express the persona pursuits and joy of life of owners. An owner fond of tea tasting can 
divide a space as a tea house to set a tea table and several cane chairs for inner tranquility and quiet thinking. An owner of calligraphy can arrange writing brushes, ink sticks, paper and ink stones, hang his/her calligraphies on the wall, arrange several pots of asparagus ferns and narcissus to foil the elegant and quiet atmosphere and the strong scholarliness.

Thirdly, soft decoration is very convenient, easy and diversified. Sometimes, several colorful cushions, pots of beautiful and stunning flowers, swaying curtains or a set of simple fabric sofa can be able to upgrade the cozy and romantic style of the room greatly. Excellent designers can use achieve the ideal space and decoration effect with less decorations, in novel and simple decoration methods. For instance, they may set a set of daily pictures on a wall to reflect the user's growth, tourism and daily experience, as the photo wall, for the aftertaste of user and appreciation of visitors, and at the same time to avoid from a large area of white space on the wall, creating the unique charm for the entire room. Or, users can change the color scheme of the interior fabrics to satisfy the demands of different seasons: in fall and winter, warm tone or thick colors can be used in textiles such as tapestry or carpets, to make the room more warm and comfortable; in spring and summer, cold tone or bright colors can be used for cooling feeling and pleasure.

\section{CONCLUSION}

From the perspective of the demands of modern interior design, soft decoration design is the result of people's upgrading requirements on living space. It is an inevitable requirement and a process to constantly enrich its design connotations that soft decoration, as a part of interior design, shall satisfy people's upgrading demands and take the prospective development trend into consideration.

\section{REFERENCES}

[1] Jian Mingmin. Soft Furnishing Designer Manual [M]. Jiangsu: Jiangsu People's Publishing House, 2011.7. 1st Version.

[2] Chang Huaisheng. Environmental Psychology and Interior Design. Beijing: China Architecture \& Building Press, 2000. 